\title{
Evidence for altered amygdala activation in schizophrenia in an adaptive emotion recognition task
}

\author{
Daniela Mier $^{\mathrm{a}, *}$, Stefanie Lis ${ }^{\mathrm{a}}$, Karina Zygrodnik ${ }^{\mathrm{b}}$, Carina Sauer ${ }^{\mathrm{a}}$, Jens Ulferts ${ }^{\mathrm{b}}$, \\ Bernd Gallhofer ${ }^{\mathrm{b}}$, Peter Kirsch ${ }^{\mathrm{a}}$ \\ a Central Institute of Mental Health, Medical Faculty Mannheim/University of Heidelberg, Mannheim, Germany \\ b Justus-Liebig University of Giessen, Giessen, Germany
}

Keywords:

Social cognition

Functional magnetic resonance imaging

Negative bias

Facial affect recognition

\begin{abstract}
A B S T R A C T
Deficits in social cognition seem to present an intermediate phenotype for schizophrenia, and are known to be associated with an altered amygdala response to faces. However, current results are heterogeneous with respect to whether this altered amygdala response in schizophrenia is hypoactive or hyperactive in nature. The present study used functional magnetic resonance imaging to investigate emotion-specific amygdala activation in schizophrenia using a novel adaptive emotion recognition paradigm. Participants comprised 11 schizophrenia outpatients and 16 healthy controls who viewed face stimuli expressing emotions of anger, fear, happiness, and disgust, as well as neutral expressions. The adaptive emotion recognition approach allows the assessment of group differences in both emotion recognition performance and associated neuronal activity while also ensuring a comparable number of correctly recognized emotions between groups. Schizophrenia participants were slower and had a negative bias in emotion recognition. In addition, they showed reduced differential activation during recognition of emotional compared with neutral expressions. Correlation analyses revealed an association of a negative bias with amygdala activation for neutral facial expressions that was specific to the patient group. We replicated previous findings of affected emotion recognition in schizophrenia. Furthermore, we demonstrated that altered amygdala activation in the patient group was associated with the occurrence of a negative bias. These results provide further evidence for impaired social cognition in schizophrenia and point to a central role of the amygdala in negative misperceptions of facial stimuli in schizophrenia.
\end{abstract}

\section{Introduction}

Patients with schizophrenia suffer from severe deficits in social cognition. These social cognitions, as defined by Brothers (1990), refer to the processing of information that leads to accurate perception of the dispositions and intentions of others. One important social cognitive function is emotion recognition, which is a necessary prerequisite for the understanding of others' intentions (Mier et al., 2010a). It has been shown that deficits in emotion and mental state recognition in schizophrenia are related to psychopathology and can affect quality of life and social outcome (Brüne, 2005; Couture et al., 2006). On the neuronal level, these deficits are reflected in atypical activation of the amygdala, which is one of the core structures used in the processing of social stimuli (Adolphs and Spezio, 2006). While many studies have demonstrated atypical amygdala functioning in schizophrenia, results are mixed with respect to whether the

\footnotetext{
* Correspondence to: Department of Clinical Psychology, Central Institute of Mental Health, J5, 68159 Mannheim, Germany. Tel.: + 49621 17036512; fax: + 4962117036505 .

E-mail address: Daniela.Mier@zi-mannheim.de (D. Mier).
}

amygdala is hyporesponsive (Li et al., 2010) or hyperresponsive (Holt et al., 2006a; Mier et al., 2010b) towards social stimuli.

Emotion recognition deficits in schizophrenia have been documented for decades (for reviews, see Edwards et al., 2002; Kohler et al., 2010). However, these deficits occur not only in periods of acute psychosis (Malik et al., 2010) and chronic schizophrenia (Mueser et al., 1996), but also in subjects at high risk of schizophrenia (Amminger et al., 2012) and in relatives of schizophrenia patients (Erol et al., 2010). Hence, it has been recently postulated that aberrant social cognition can be viewed as an intermediate phenotype of schizophrenia (Derntl and Habel, 2011).

While findings from behavioral studies of schizophrenia clearly point to deficits in social cognition, results from studies investigating the neurobiological correlates of social cognition, and particularly emotion recognition in schizophrenia, are rather mixed (although it should be mentioned that findings from behavioral studies also differ with regard to which emotions are most difficult to recognize for schizophrenia patients) (e.g., Kohler et al., 2003; Silver et al., 2009). Meta-analyses point to the amygdala as the structure that shows the most systematic differences between schizophrenia patients and healthy controls during emotion recognition 
(Li et al., 2010; Anticevic et al., 2012; Taylor et al., 2012). However, while most of the early studies found hypoactivation of the amygdala and other structures in schizophrenia (see meta-analyses by Li et al., 2010; Anticevic et al., 2012; Taylor et al., 2012; Delvecchio et al., 2013), more recently a growing number of studies in schizophrenia have reported hyperactivation of several emotion-related brain areas, including the amygdala (Holt et al., 2006a; Seiferth et al., 2008; Mier et al., 2010b). Such heterogeneity of results (concerning hypoactivity versus hyperactivity of the amygdala) might be due to methodological differences between the studies, including (but not limited to) the emotions that have to be recognized, as well as the subsequent analysis of task effects.

First of all, there is evidence that hypoactivation in response to emotional expressions in schizophrenia occurs only for specific types of emotions. While most of the studies examining general emotion recognition abilities found hypoactivation (Li et al., 2010), studies comparing activation in response to neutral facial expressions found hyperactivation of the amygdala in schizophrenia patients (Holt et al., 2006a; Mier et al., 2010b). Going along with findings of hyperactivation of the amygdala in the processing of neutral facial expressions, several studies demonstrated altered salience attribution to neutral stimuli in schizophrenia (Holt et al., 2006b), as well as a negative bias (Premkumar et al., 2008); that is, patients are more likely than healthy control subjects to perceive negative emotions in neutral facial expressions. Hall and colleagues (Hall et al., 2008) demonstrated amygdala hypoactivation in a sample of schizophrenia patients in response to fearful faces, but only when contrasting the activation with activation in response to neutral facial expressions. These results by Hall and colleagues are in agreement with a recent meta-analysis by Anticevic and colleagues (Anticevic et al., 2012), showing amygdala hypoactivity for negative emotions in schizophrenia, when activation was contrasted with neutral emotions. Hence, there seems to be a difference in the processing of emotional and neutral facial expressions between schizophrenia patients and healthy controls, suggesting that a separate investigation of brain response to neutral and emotional facial expressions in schizophrenia might be beneficial (Mier et al., 2010b).

Two additional factors that have important implications for the strength of brain activation are task difficulty and task motivation. It has been demonstrated that low performance in working memory tasks is associated with decreased activation of prefrontal areas (Callicott et al., 2003; Van Snellenberg et al., 2006). Comparable evidence can be found for emotional processing. Hempel and colleagues showed that activation in prefrontal areas increases when emotion recognition is more difficult (Hempel et al., 2003). These prefrontal areas in turn have a modulatory influence on the amygdala (Stein et al., 2007a), and it was shown that when a task has a higher cognitive demand, task-irrelevant emotional stimuli have a weaker effect on amygdala activation (Pessoa et al., 2005; Jasinska et al., 2012), and that amygdala activation is stronger when emotion processing is less explicit (Hariri et al., 2000). Similar to these results, we demonstrated reduced amygdala activation in schizophrenia patients with increasing task demand and decreasing performance in a social-cognitive task (Mier et al., 2010b). Moreover, a motivational deficit, associated with reduced activation in the ventral striatum has been repeatedly demonstrated in schizophrenia (Esslinger et al., 2012; Nielsen et al., 2012; Gradin et al., 2013). Hence, there is evidence that task difficulty and motivation can have an indirect or even a direct influence on activation of limbic structures. Since patients with schizophrenia are impaired in emotion recognition, i.e., the task is more difficult for them, one could assume that this impairment is also reflected in reduced amygdala activation. Hence, in attempts to determine whether schizophrenia is associated with altered amygdala activation during emotion recognition, comparable task difficulty has to be established for the patients and the control group. In other words, differences in task difficulty between groups have to be avoided because they could lead to altered amygdala activation in various ways that are not necessarily caused by a biological emotion recognition deficit.

Here, we present a study that tests the ability to recognize positive and negative emotions, as well as neutral facial expressions, in schizophrenia, using an adaptive emotion recognition paradigm. An adaptive design has the advantage that the intensity of the to-be-recognized emotion is adjusted to each subject's ability to recognize emotions, allowing for comparable levels of subjective difficulty (in terms of the number of correct responses) between subjects and groups. By adjusting the task difficulty based on subject ability, we can also avoid confounds in brain activation that are due to performance differences between groups. We hypothesized that in comparison to a healthy control group, schizophrenia patients would have prolonged reaction times and show a necessity for higher emotional intensities for accurate emotion recognition, as well as a negative bias. In addition, we hypothesized that we would find amygdala hyperactivation in schizophrenia subjects for the recognition of neutral facial expressions, but not for the recognition of emotional expressions. Lastly, we hypothesized that the strength of amygdala activation for neutral facial expressions would be correlated with the amount of negative bias.

\section{Methods}

\subsection{Subjects}

Participants comprised 12 schizophrenia outpatients and 16 healthy control subjects. One schizophrenia patient was excluded from analyses because he recognized none of the faces with a fearful expression correctly. Groups were matched for age, gender, fluid and crystalline intelligence, and education. With the exception of one left-handed patient, all participants were right-handed. All participants (as well as all facial stimuli for the emotion recognition task) were Caucasian. Healthy controls were excluded if they indicated having a current or lifetime psychiatric disorder, as assessed with the German screening interview MINI-DIPS (Margraf, 1994). Moreover, control subjects were also excluded if they reported having any first degree relatives with a psychiatric disorder (self-report). All patients met criteria for a DSM-IV diagnosis of schizophrenia and were on stable doses of antipsychotic medication. To assess current schizophrenic psychopathology in the patient group, Schedule for the Assessment of Positive Symptoms (SAPS) (Andreasen, 1984) and the Schedule for the Assessment of Negative Symptoms (SANS) (Andreasen, 1983) were applied by an experienced clinical psychologist (D.M.). Patients were low on positive symptoms, as well as on negative symptoms. Table 1 presents demographic information, chlorpromazine-equivalent antipsychotic dosage (Andreasen et al., 2010; Gardner et al., 2010), and psychopathology (see Supplementary Table 1 for detailed information on medication for each of the patients). All subjects fulfilled inclusion criteria for magnetic resonance imaging. Before participation, written informed consent was acquired from all subjects. The study was approved by the local ethics board of the University of Giessen, School of Medicine, and conducted in agreement with the declaration of Helsinki.

\subsection{Stimuli}

Facial stimuli with neutral, angry, happy, fearful, or disgusted expressions were presented. To generate facial stimuli with graded emotional intensities, pictures from eight people (four females) were subjected to a morphing procedure. A morphing program was used to prepare pictures with $20 \%, 40 \%, 60 \%$, and $80 \%$ intensity of the emotion by superimposing the neutral expression of each person with their emotional expression, resulting in one neutral picture for each person and five pictures (the original emotion and four generated ones) for each of the emotions. Next, these pictures were shown to 93 university students for emotion recognition (Mier et al., 2010a). Based on their ratings, five intensity levels of the emotions $(20 \%, 40 \%, 60 \%, 80 \%$, and $100 \%$ ) were reproduced (which were not necessarily identical to the grading of the morphing program). These stimuli were used to adjust the difficulty of emotion recognition to achieve comparable average recognition rates for all subjects. The aim of this process was not to create finetuned stimuli with which, for example, an emotion-detection threshold could be defined, but rather to generate enough stimuli that varied in difficulty to apply an adaptive emotion recognition task. 
Table 1

Clinical and sociodemographic variables. Note: S.D.=standard deviation. Antipsychotic medication is reported in chlorpromazine equivalents.

\begin{tabular}{llll}
\hline & Schizophrenia patients & Healthy controls & Significance \\
\hline Gender (males) & $64 \%$ & $68 \%$ & $\chi, p=1.0, n . s$. \\
Age (mean, S.D.) & 32.45 years (7.66) & 34.50 years (6.47) & $p=0.46, n . s$. \\
Education (mean, S.D.) & 14.73 years (1.62) & 14.00 years (1.63) & $p=0.27, n . s$. \\
Crystalline intelligence (mean, S.D.) & $113.45(14.61)$ & $114.94(12.06)$ & $p=0.78, n . s$. \\
Fluid Intelligence (mean, S.D.) & $117.10(19.57)$ & $119.50(17.63)$ & \\
SAPS (mean, S.D.) & $1.45(1.63)$ & & \\
SANS (mean, S.D.) & $6.59(2.13)$ & & \\
Antipsychotics (mean, S.D.) & $472.56(269.06)$ & & \\
Antidepressants & $37 \%$ & & \\
Anticonvulsants & $27 \%$ & & \\
Age of onset (mean, S.D.) & 23 years (6.91) & \\
Duration of illness (mean, S.D.) & 10.18 years (8.92) & \\
Number of episodes (mean, S.D.) & $4.91(4.13)$ & & \\
\hline
\end{tabular}

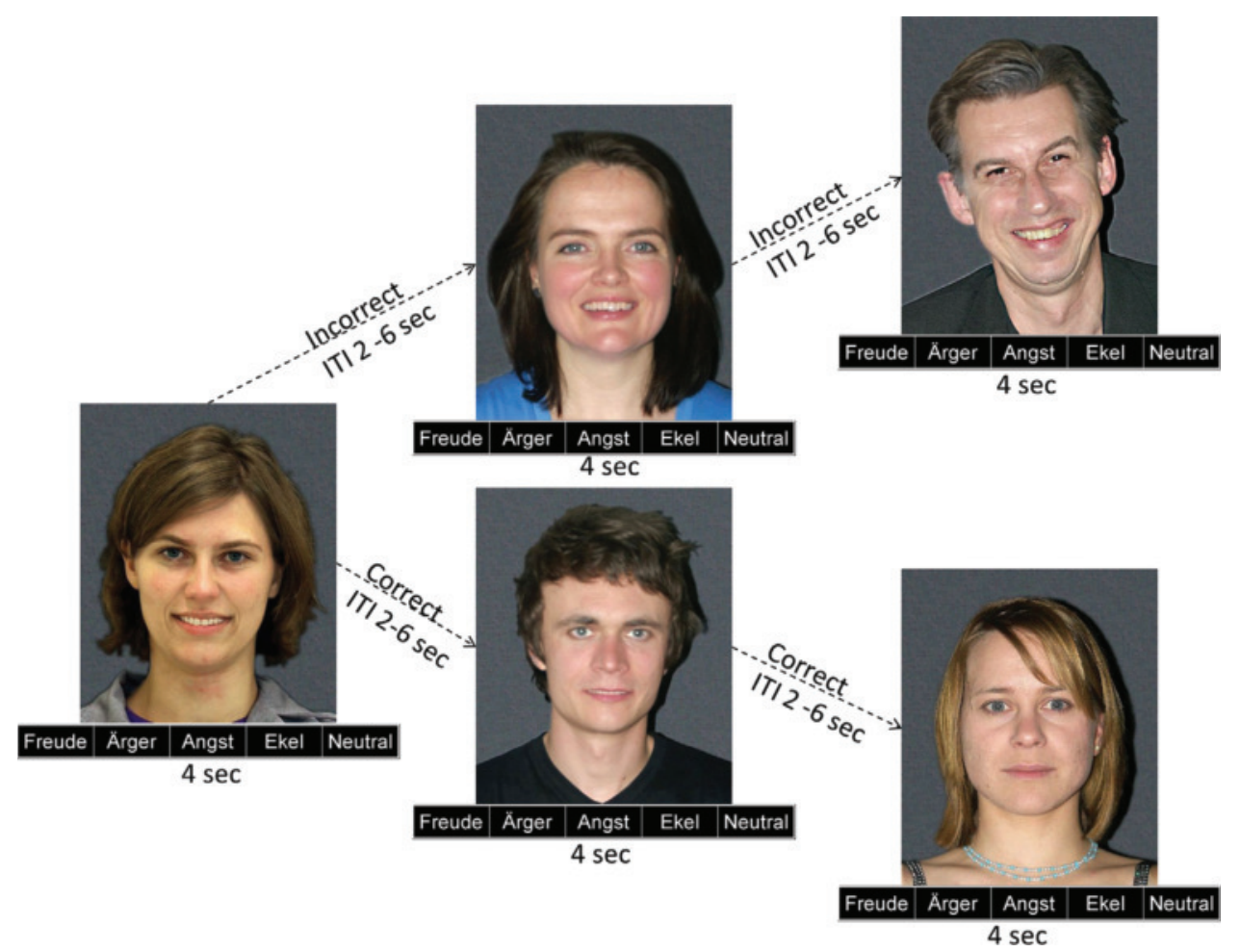

Fig. 1. Adaptive emotion recognition design, illustrated for the emotion happiness. Subjects started for each emotion with a medium emotional intensity. After the emotion had been recognized correctly, the next trial with the emotion had a lower emotional intensity; when the emotion was not recognized correctly, the next trial with the emotion had a higher emotional intensity. The pictures were shown with the response categories: Happiness, anger, fear, disgust and neutral below. Note: Happiness = Freude, Anger $=$ Ärger, Fear $=$ Angst, Disgust $=$ Ekel and Neutral $=$ Neutral, ITI $=$ Inter-trial interval.

\subsection{Experimental design}

Subjects were shown face pictures one at a time simultaneously with the five emotion response options: Happiness, Anger, Fear, Disgust, and Neutral. They were instructed to identify the emotion as fast as possible by button press. All subjects started with faces of medium emotional intensity for each of the emotions. Depending on the subject's performance, the intensity of the emotional expression in the next trial was adjusted. Across the experiment, this procedure resulted in comparable subjective difficulty of the task, but possible objective differences in reaction times and average intensity of the emotion. Since the neutral facial expressions were not adapted to the subjects' performance, the number of incorrect trials served as indicator of emotion recognition deficits.

Emotions were presented in a pseudorandom order, and the adaptive process was applied for each emotion separately. In case of participants showing a floor or a ceiling effect for a specific emotion, stimuli of the $100 \%$, respectively $20 \%$ intensity had to be presented repeatedly. There were 20 trials for each emotion, resulting in a total of 100 trials. Each trial had a duration of $4 \mathrm{~s}$, followed by an inter-trial interval of $4 \mathrm{~s}$ (with a jitter between 2 and $6 \mathrm{~s}$ ). Responses were given by pressing one of five buttons of a self-designed response box, each assigned to a particular emotion. Fig. 1 illustrates the experimental paradigm with the emotion happiness as an example.

\subsection{Functional magnetic resonance imaging}

\subsubsection{Data acquisition}

Functional magnetic resonance data were collected with a $1.5 \mathrm{~T}$ General Electric (Milwaukee, IL) whole body scanner. A total of 279 volumes were collected with an axial T2*-weighted echo planar imaging (EPI) sequence (30 slices, slice thickness $5 \mathrm{~mm}$, repetition time 3000 , echo time 50 , flip angle $90^{\circ}$, field of view $220,64 \times 64$ matrix). Slices were adjusted to the anterior commissure-posterior commissure (AC-PC line), and acquired in an interleaved order. Prior to the echo planar sequence, a 3D-T1-weighted anatomical scan with 172 slices (voxel size $0.94 \times 0.94 \times 0.94 \mathrm{~mm}$ ) was acquired. 


\subsubsection{Data analyses}

Functional imaging data were analyzed with Statistical Parametric Mapping 8 (SPM8). Prior to analyses, data were pre-processed, applying slice time correction, realignment, and normalization to the standard EPI template and smoothing with a $10-\mathrm{mm}$ isotropic Gaussian kernel. Data were subjected to a first level analysis modeling the fit of the data with the emotion recognition conditions. For this purpose, five regressors with the onsets of the emotion recognition trials were defined (one regressor for all trials of a certain emotion, independent of whether participants recognized the emotion in a given trial correctly). In addition, information about movement-related variance, resulting from the realignment (six regressors) were included as covariates. Regressors were folded with a stick function and convolved with a synthetic hemodynamic response function. Group effects were analyzed in second level random effects analyses, containing the information from the five regressors of interest from the first level. The main effect of group and the interaction between group and condition were analyzed by applying a 2 (group) $\times 5$ (emotion) mixed effects model. Post hoc comparisons of activation between groups within the emotion recognition conditions were conducted with two-sample $t$-tests. In addition, exploratory correlation analyses between brain activation and behavior were achieved by applying regression analyses.

The significance threshold was set to $p<0.05$, family-wise error-corrected for whole brain analyses. Cluster size threshold was set to $k \geq 5$. Masks for amygdala region of interest (ROI) analyses were taken from the Wake Forest University Pickatlas (http://fmri.wfubmc.edu). Significance threshold for the ROI analyses was set to $p<0.05$, small volume (sv) corrected, again with a cluster size threshold of $k \geq 5$. Results are reported in Montreal Neurological Institute coordinates.

Behavioral data were analyzed with SPSS V20. Number of correct trials, as well as reaction times of correct trials, were analyzed with 2 (group) $\times 5$ (emotion) repeated measures analyses of variance (ANOVAs). Intensities were analyzed with a 2 (group) $\times 4$ (emotion) repeated measures ANOVA. One-sample $t$-tests were applied for post hoc comparisons of intensities and reaction times. Two-sample $t$-tests were used for group comparisons for the number of correct responses in trials with neutral facial expressions, for post hoc group comparisons of reaction times, as well as the intensity of the emotions. In addition, a possible negative response bias was analyzed by calculating a compound score of the average number of negative misclassifications (neutral expressions classified as negative and happy expressions classified as negative) and compared between groups with a two-sample $t$-test. Lastly, to investigate associations between amygdala activation and behavior, as well as psychopathology, we extracted the time course within left and right amygdala mask (for the contrasts: happy, angry, fear, disgust and neutral, as well as for the contrasts: happy $>$ neutral, angry $>$ neutral, fearful $>$ neutral, and disgust $>$ neutral) and correlated these with the mentioned parameters in SPSS.

\section{Results}

\subsection{Behavioral results}

The average number of correctly recognized emotions was 9.38 $(46.9 \%$ of the trials, S.D. $=0.78)$, demonstrating that subjects indeed solved the task in approximately half of the trials, proving suitability of the adaptive algorithm. Comparison of the number of correct emotion trials between the groups showed the groups did not differ significantly $(F(25 ; 1)=0.34, p=0.57 ; f=0.13)$, demonstrating that the adaptive algorithm led to comparable subjective task difficulty for both groups. Between-group comparison of the trials with neutral facial expressions showed that schizophrenia

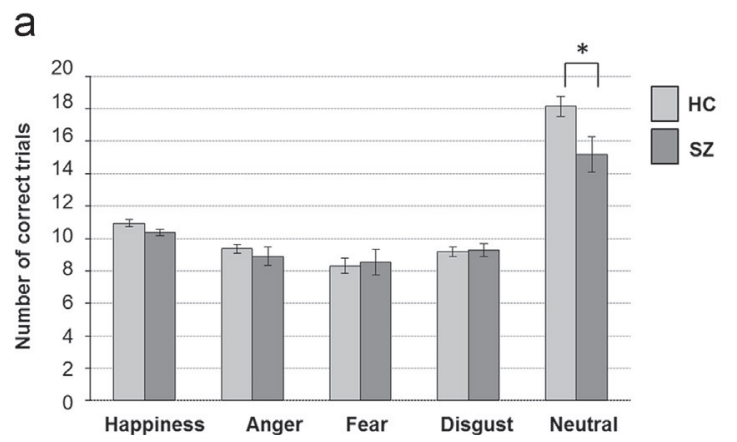

subjects made significantly more errors in the recognition of neutral facial expressions $(t(24)=2.39, p=0.026 ; f=0.92)$. Fig. 2a presents correct responses for both groups. Analysis of the intensity of the emotion trials showed a significant main effect of emotion $(F(3 ; 22)=31.85, p<0.001 ; f=3.41)$, but not of group, nor an interaction between group and emotion type. Subjects needed lower intensities for the recognition of happiness than for the recognition of the negatively valenced facial expressions (all $p s<0.001$, all $t s>9.52$ ). This effect remained significant after correcting for multiple testing. Intensity levels of the negative emotions did not differ significantly (all $p s>0.72$, all $t s<0.36$ ). Analysis of the reaction times showed a significant main effect of emotion $(F(4 ; 22)=51.85, p<0.001 ; f=1.43)$ and of group $(F(1 ; 24)=13.53, p=0.001 ; f=0.75)$, but no significant interaction between group and emotion (see Fig. 2b). Post hoc comparisons showed that reaction times differed between the emotions, except between disgust and neutral facial expressions (all $p s<0.05$, all ts $>2.38$ with fear $>$ anger $>$ happiness $>$ neutral=disgust). The comparisons between reaction times remained significant after correction for multiple testing, except for the difference between anger and disgust. In addition, it was revealed that patients with schizophrenia were significantly slower than healthy controls in recognizing happiness, anger, fear and neutral facial expression, but not disgust (all $p s<0.05$, all $t s>2.13$ ). However, when correcting for multiple testing only the reaction time differences between groups for the recognition of happiness and neutral remained significant.

In addition, we investigated the pattern of errors to see whether schizophrenia patients showed a negative bias in the perception of happy and neutral facial expressions. Group comparison of the average number of 'negative' misclassifications showed an enhanced negative bias in schizophrenia $(t(25)=1.83, p=0.04$; $f=0.68$ ).

\subsection{Functional brain imaging results}

Comparison of groups revealed hypoactivation in schizophrenia subjects in several inferior parietal and temporal areas, in the inferior prefrontal gyrus, anterior cingulate and cerebellum (Table 2). The reverse contrast revealed increased activation in occipital areas (Table 2). In addition, ROI analysis for the amygdala showed significant hypoactivation in the left amygdala in the schizophrenia subjects (coordinates: $-27-4-17, t=3.15, p=0.010$ sv-corrected, $k=38, f=0.61)$.

Since we were interested in differences in amygdala activation between groups depending on the type of the presented emotion, a directed interaction analysis was conducted, testing the assumption that schizophrenia subjects have more activation when the target is a neutral face and healthy controls when the target is an emotional face. Whole brain analysis showed a significant interaction in the supramarginal and superior temporal gyrus (Table 2).

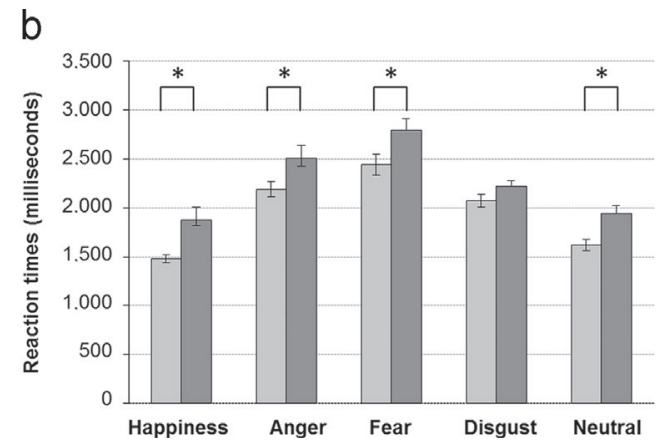

Fig. 2. Emotion recognition performance for both groups. (a) Number of correctly recognized trials, separately for the emotions. (b) Reaction times, separately for the emotions. Note: Significant differences between groups are indicated by stars, SZ=schizophrenia patients, HC=healthy controls, displayed are averages with standard deviation bars. 
In addition, ROI analysis revealed a significant interaction effect in the left amygdala (coordinates: $-18-7-23, t=3.27, p=0.007$, $k=44, f=0.64$; Fig. 3 ).

Post hoc whole brain group comparisons showed significantly reduced activation in a cluster in the cerebellum for the schizophrenia patients while recognizing fearful facial expressions (coordinates: $24-88-29, t=6.38, p=0.009, k=7$ ). No other group differences at the given significance level were revealed. Post hoc group amygdala ROI comparisons revealed only a significant hypoactivation in the left amygdala for happiness in the patient group (coordinates: $-272-20, t=3.35, p=0.014, k=20$, $f=0.65$ ).

\section{Table 2}

Functional brain imaging results for the main effects of group and the group by emotion interaction $(p<0.05$ FWE-corrected). Note: HCs $=$ Healthy controls, SZs $=$ Schizophrenia subjects. Subcluster peaks are inserted.

\begin{tabular}{|c|c|c|c|c|c|c|}
\hline \multirow{2}{*}{$\begin{array}{l}\text { Main effect HCs }>S Z s \\
\text { Area }\end{array}$} & \multirow[t]{2}{*}{$\mathrm{BA}$} & \multirow[t]{2}{*}{ Cluster } & \multicolumn{3}{|c|}{ MNI } & \multirow[t]{2}{*}{$T$-value } \\
\hline & & & $x$ & $y$ & $z$ & \\
\hline Inferior Parietal Lobule & 40 & 216 & 45 & -55 & 58 & 8.27 \\
\hline Inferior Parietal Lobule & 39 & & 48 & -67 & 40 & 5.68 \\
\hline Cerebellum & & 24 & 27 & -88 & -29 & 6.38 \\
\hline Insula & 13 & 194 & -30 & 17 & -5 & 6.27 \\
\hline Superior Temporal Gyrus & 38 & & -51 & 17 & -17 & 6.16 \\
\hline Fusiform Gyrus & 20 & 96 & -45 & -25 & -17 & 6.23 \\
\hline Middle Temporal Gyrus & 22 & & -54 & -40 & -5 & 5.67 \\
\hline Inferior Frontal Gyrus & 46 & 78 & -54 & 29 & 16 & 5.97 \\
\hline Inferior Frontal Gyrus & 47 & 264 & 45 & 20 & -14 & 5.94 \\
\hline Insula & 13 & & 33 & 23 & 1 & 5.74 \\
\hline Inferior Frontal Gyrus & 45 & & 60 & 20 & 22 & 5.73 \\
\hline Inferior Parietal Lobule & 40 & 142 & -48 & -49 & 52 & 5.86 \\
\hline Superior Parietal Lobule & 7 & & -42 & -67 & 52 & 5.71 \\
\hline Inferior Parietal Lobule & 40 & & -45 & -55 & 58 & 5.33 \\
\hline Middle Temporal Gyrus & 21 & 13 & 60 & -25 & -17 & 5.52 \\
\hline Transverse Temporal Gyrus & 41 & 18 & -51 & -19 & 10 & 5.48 \\
\hline Cerebellum & & 42 & -36 & -70 & -32 & 5.25 \\
\hline Anterior Cingulate & 32 & 33 & 21 & 23 & 22 & 5.02 \\
\hline Anterior Cingulate & 32 & & 12 & 26 & 25 & 4.72 \\
\hline Cerebellum & & 10 & -18 & -49 & -26 & 4.77 \\
\hline Postcentral Gyrus & 5 & 11 & -21 & -46 & 70 & 4.76 \\
\hline \multicolumn{7}{|l|}{$\begin{array}{l}\text { Main effect SZs > HCs } \\
\text { Area }\end{array}$} \\
\hline Cuneus & 18 & 16 & -3 & -100 & 7 & 5.01 \\
\hline Middle Occipital Gyrus & 19 & 8 & -45 & -82 & -2 & 4.82 \\
\hline Cuneus & 17 & 7 & 18 & -94 & 4 & 4.74 \\
\hline \multicolumn{7}{|l|}{$\begin{array}{l}\text { Group } \times \text { Condition interaction } \\
\text { Area }\end{array}$} \\
\hline Supramarginal gyrus & 40 & 23 & 45 & -55 & 58 & 5.68 \\
\hline Superior temporal gyrus & 22 & 6 & -54 & -40 & -5 & 4.64 \\
\hline
\end{tabular}

In comparisons of differential activation between groups (emotions $>$ neutral), there was significantly reduced amygdala activation in the patient group for fear (left coordinates: -18 $-4-20, t=2.93, p=0.032$ sv-corrected, $k=30, f=0.56$; right coordinates: $24-4-17, t=3.01, p=0.026, k=43, f=0.61$ ), for happiness (left coordinates: $-24-4-20, t=3.18, p=0.019$, $k=36, f=0.61$ ) and for anger (left coordinates: $-21-4-26$, $t=3.31, p=0.014, k=39, f=0.60$; and a trend in right amygdala, coordinates: $242-20, t=2.34, p=0.086, k=16, f=0.45)$. No further significant differences in amygdala activation were found between groups.

To explore the relationship between amygdala activation and the existence of a negative bias, correlational analyses were conducted. In analyses of the correlations between the contrasts of emotional versus neutral facial expressions, a negative significant correlation of left amygdala activation for fear $>$ neutral $(r=-0.69, p=0.017)$ occurred in the patient group, indicating more negative bias and less increase of left amygdala activation for the recognition of fear in comparison to the recognition of a neutral facial expression (Fig. 4b). In addition, a positive significant correlation between negative bias and right amygdala activation for neutral facial expressions was revealed $(r=0.65, p=0.03)$ in the patient group (Fig. 4a). Furthermore, we were interested in the relationship between amygdala activation and the required intensity of the emotion, as well as amygdala activation and reaction times. Here, we found that higher intensities of anger were needed when patients had less increase of amygdala activation (anger > neutral) for anger (left amygdala: $r=-0.60, p=0.051$; right amygdala: $r=-0.73, p=0.01$; Fig. $4 \mathrm{~d}$ ), and for happiness recognition (happiness $>$ neutral) in right amygdala $(r=-0.80$, $p=0.003$; Fig. 4c). None of the correlations between intensity and amygdala activation was significant (when the response to neutral facial expressions was not subtracted). Only one correlation was significant for the control group. Reaction times for the recognition of happy faces were negatively associated with the increase of amygdala activation for happiness in comparison to neutral facial expressions $(r=-0.56, p=0.024)$. However, none of the reported correlations survived correction for multiple testing.

Furthermore, correlation analyses between schizophrenic psychopathology and amygdala activation showed that positive pathology (SAPS total score) was associated with an enhanced increase in amygdala activation during anger recognition in comparison to the recognition of neutral facial expressions (left amygdala: $r=0.63, p=0.039$; right amygdala: $r=0.88, p<0.001$ ), but negative pathology (SANS total score) with reduced activation increase (left amygdala: $r=-0.68, p=0.021$; right amygdala: a

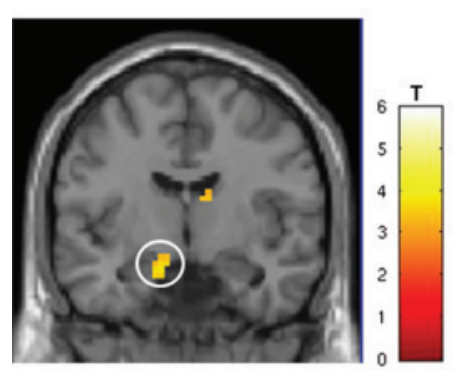

b

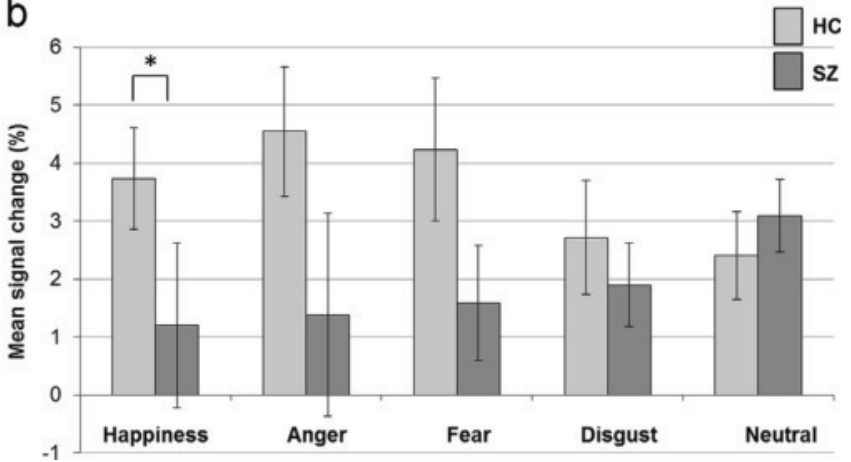

Fig. 3. Left amygdala activation, with an interaction between facial expression and group. (a) Group by emotion interaction in left amygdala ( $p<0.001$ uncorrected for display purposes; the left amygdala is highlighted by a white circle). (b) Mean signal change in the left amygdala, depending on group and facial expression (displayed is the mean signal change within the left amygdala region of interest with standard deviation bars). Note: Significant differences between groups are indicated by stars, $\mathrm{SZ}=$ schizophrenia patients, $\mathrm{HC}=$ healthy controls. 


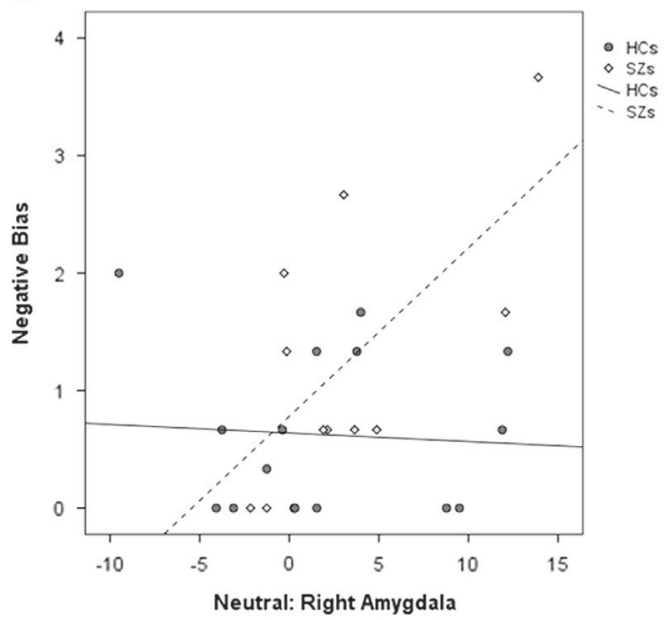

C

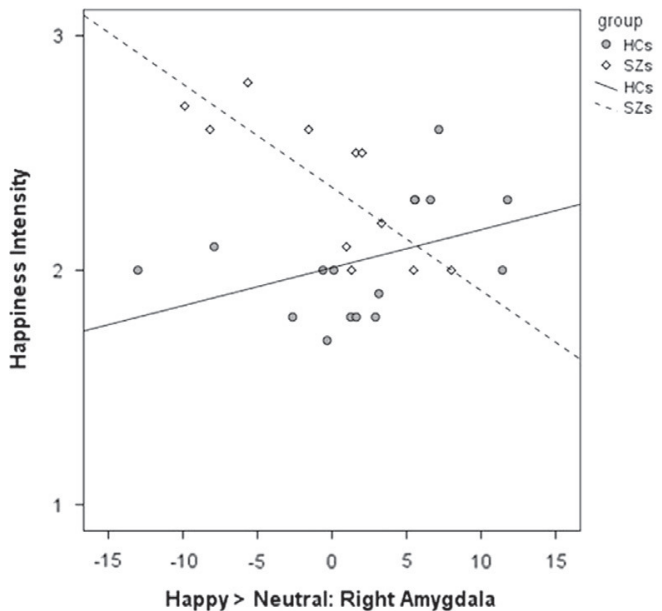

b

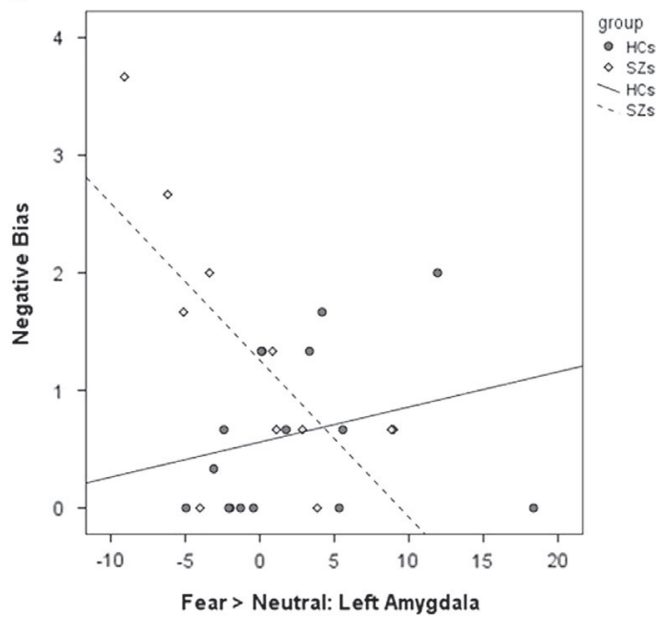

d

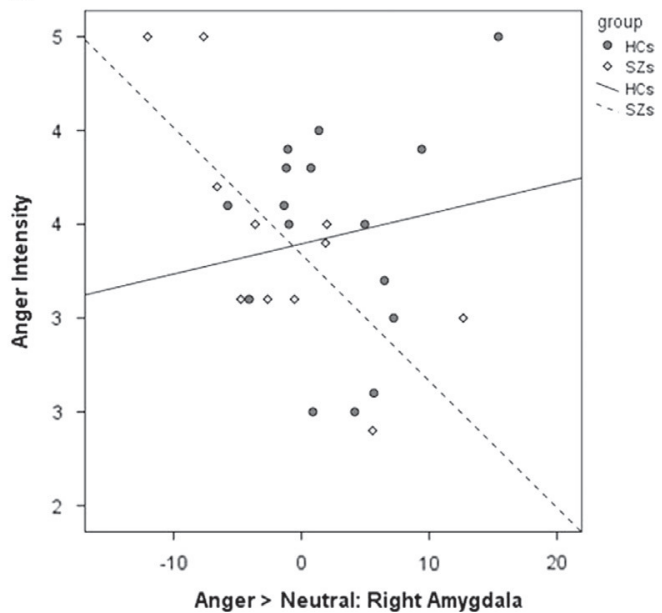

Fig. 4. Correlations between brain activation and performance. (a) Correlation between right amygdala activation for neutral facial expression and negative bias. (b) Correlation between left amygdala activation in the contrast fear $>$ neutral and negative bias. (c) Correlation between right amygdala activation in the contrast happiness > neutral and required intensity for happiness recognition. (d) Correlation between right amygdala activation in the contrast anger $>$ neutral and required intensity for happiness recognition. Note: signal change from left and right amygdala masks was extracted and correlated with behavioral measures within SPSS V20; all displayed correlations are significant for the patient group, but not for the healthy control group. SZs=schizophrenia patients, HCs=healthy controls.

$r=-0.52, p=0.11, n . s$.$) . In addition, right amygdala activation$ increase in response to disgust faces was associated with more positive pathology $(r=0.72, p=0.01)$, while the opposite was true for left amygdala activation in response to happy facial expressions $(r=-0.67, p=0.02)$. No significant associations between psychopathology and behavior were revealed. In addition, no significant correlations between chlorpromazine-equivalent antipsychotic dose and brain activation were revealed. Finally, a significant association between age of onset and reaction times for the recognition of disgust $(r=-0.64, p=0.04)$, as well as for the number of psychotic episodes and reaction times for neutral facial expressions, was revealed $(r=-0.64, p=0.04)$. However, again, none of these correlations survived correction for multiple testing.

\section{Discussion}

The present study was set-up to explore the role of the amygdala in social cognition deficits in schizophrenia patients by focusing on emotion recognition related changes in amygdala activation. To avoid conditions that might confound the true group differences, we applied an adaptive emotion recognition task that would minimize the effects of motivational differences between groups due to increased difficulty of task-solving in the patient group.

Schizophrenia patients were found to be affected in their overall emotion recognition performance. This deficit was pronounced for the recognition of neutral expressions. While reaction times were generally longer in the schizophrenia group for all emotions except for disgust, recognition deficits occurred only when recognizing positive or neutral expressions; that is, schizophrenia patients showed a negative bias compared with controls, more often perceiving happy and neutral expressions as negative. This result is only partially in agreement with the literature, since most studies provide evidence for general emotion recognition deficits in schizophrenia (Edwards et al., 2002; Kohler et al., 2010), while more recent studies often demonstrate a deficit in the recognition of positive emotions (Tsoi et al., 2008; Laroi et al., 2010) and specifically a negative bias (Tsoi et al., 2008; Constant et al., 2011; Haralanova et al., 2012). One interpretation of these results is a heightened sensitivity for (negative) emotions, as has been assumed for borderline personality disorder (Lynch et al., 2006). This interpretation, however, is not further supported by the recognition pattern of negative emotional expression in the present study, since patients did not need less intensive emotional expression for the accurate perception of negative emotions. 
Interestingly, Tsoi and colleagues found not only a deficit in the recognition of happy faces in schizophrenia, but also a tendency to more liberally attribute fear and anger to all kinds of emotions (Tsoi et al., 2008). Hence, it seems that while schizophrenia subjects have a greater tendency to attribute negative emotions to neutral and/or ambiguous emotional expressions, they do not necessarily also have an increased sensitivity for those emotions. In sum, on the behavioral level, we find evidence for longer response times in schizophrenia subjects, a deficit in the recognition of neutral and positive emotional expressions, and a negative bias that might point to a more liberal threshold to attribute negative emotions.

In terms of brain activation, the results give evidence for an impaired social cognitive network in schizophrenia subjects. Schizophrenia subjects had reduced activation in several areas, including the amygdala, temporo-parietal junction and inferior prefrontal gyrus. Furthermore, a group by emotion interaction occurred in the temporo-parietal junction. These areas have been associated with the human mirror neuron system (Rizzolatti and Craighero, 2004) and are assumed to support embodied simulation (Gallese, 2007). On the other hand, schizophrenia patients had increased activation in the cuneus bilaterally and in the left middle occipital gyrus. This hyperactivation is in agreement with a recent meta-analysis, suggesting hyperactivation in visual areas during emotion perception in schizophrenia (Taylor et al., 2012). Together, the pattern of hypoactivation as well as hyperactivation suggests a less automatic (i.e., less embodied) perception of facial expressions in schizophrenia that could be related to increased demands on visual processing.

Specifically for the amygdala, a group by emotion interaction was found, indicating reduced amygdala activation in schizophrenia patients while recognizing emotions, but not while recognizing neutral facial expressions. Post hoc comparisons between groups were not significant, except for left amygdala activation for happiness. However, when activation in response to emotional expressions was contrasted with the response to neutral expressions, reduced differential amygdala activation was found for fear, anger and happiness in the patient sample. These results are partially in agreement with our hypotheses and with recent metaanalyses of amygdala activation, suggesting reduced differential amygdala activation in schizophrenia (Li et al., 2010; Anticevic et al., 2012; Taylor et al., 2012), and have several implications. First of all, they support findings demonstrating that the amygdala in schizophrenia subjects is not generally hypoactive in response to facial stimuli (Kosaka et al., 2002; Holt et al., 2006a; Mier et al., 2010b; Rauch et al., 2010; Sachs et al., 2012). Second, they agree with studies showing a special relevance of the processing of neutral (Habel et al., 2010; Mier et al., 2010b; Pinkham et al., 2011) as well as positive facial information (Kosaka et al., 2002) in schizophrenia. Third, they suggest that neutral facial expressions used as a control condition might (artificially) augment group differences in response to emotional expressions (Anticevic et al., 2012).

In line with the above-mentioned studies, correlation analyses revealed an association between amygdala activation and negative bias that was specific for the patient group. Enhanced amygdala activation in response to neutral facial expressions was associated with more negative bias, suggesting a relationship between increased amygdala activation in response to neutral facial expressions and the misattributions of negative emotions. This conclusion is also supported by the findings of a negative association between amygdala activation and required emotional intensity for happiness and anger, as well as by an association of positive pathology and amygdala activation for neutral facial expressions. However, none of these correlations survived the significance threshold for multiple testing.

We have argued before that hyperactivation of the amygdala in response to neutral facial expressions in schizophrenia patients might be a trait marker for schizophrenia, making patients prone to positive pathology (Mier et al., 2010b). However, it has been suggested that altered amygdala activation is not an intermediate phenotype (or a trait marker) of schizophrenia, but dependent on current pathology (Rasetti et al., 2009). Our need to understand whether and when schizophrenia is associated with a hyperactive or a hypoactive amygdala is not just of an academic nature, but has significant implications for our understanding of the schizophrenic pathology and necessary treatment. Our results support the assumption that schizophrenia psychopathology has a differential relationship with amygdala activation in response to neutral and emotional facial expression recognition. Patients with positive pathology can show increased amygdala activation in response to neutral facial expressions, for example, as shown by Pinkham and colleagues (Pinkham et al., 2008), but it might be that negative pathology, especially flat affect, is associated with reduced amygdala activation in response to emotional facial expressions. While amygdala hypoactivation has been linked with psychopathy (Kiehl et al., 2001) and anhedonia (Keedwell et al., 2005; Dowd and Barch, 2010), amygdala hyperactivation has been linked with borderline personality disorder (Mier et al., 2013), anxiety (Stein et al., 2007b), and reduced emotion regulation (Drabant et al., 2009). Hence, it is essential to know whether treatment should focus on enhancing emotional reactivity or reducing anxiety to social stimuli in order to subsequently enhance social-cognitive abilities.

For these reasons, studies with a sufficient number of schizophrenia patients are needed that would allow the application of regression analyses and further investigation into the effects of amygdala hyperactivation on behavior and subsequently psychopathology. In addition, based on the small patient sample sizes in the present study, correlational analyses bear the risk of falsenegative findings. Bigger samples would also provide the added benefit of more reliable interpretation of laterality effects of group differences. Furthermore, we investigated only schizophrenia outpatients who were low in positive psychopathology, which prevents us from drawing conclusions about patients suffering from acute psychosis or patients with chronic positive psychopathology. Lastly, future studies should ask subjects for a rating of their experienced difficulty in performing the emotion recognition task as a further measure of the suitability of the adaptive algorithm. Hence, replication studies with bigger samples and a broader range of schizophrenic pathology are needed, which would likely reveal even stronger group differences, enhance the reliability of correlational analyses, and allow for the assessment of further variables of interest.

In conclusion, the present study supports the notion of socialcognitive deficits representing an intermediate phenotype for schizophrenia. The adaptive emotion recognition paradigm is suitable to elicit activation in the amygdala and to investigate alterations in emotion recognition performance and associated neural activation in schizophrenia. Schizophrenia subjects seem to have different deficits in the recognition of emotions and the recognition of a neutral expression as neutral that is reflected in performance and altered amygdala activation in comparison to healthy controls. Future studies with bigger samples should investigate the relationship with schizophrenia psychopathology, in order to guide adequate therapeutic strategies depending on the disease state. 


\section{References}

Adolphs, R., Spezio, M., 2006. Role of the amygdala in processing visual social stimuli. Progess in Brain Research 156, 363-378.

Amminger, G.P., Schafer, M.R., Papageorgiou, K., Klier, C.M., Schlogelhofer, M. Mossaheb, N., Werneck-Rohrer, S., Nelson, B., McGorry, P.D., 2012. Emotion recognition in individuals at clinical high-risk for schizophrenia. Schizophrenia Bulletin 38, 1030-1039.

Andreasen, N.C., 1983. The Scale for the Assessment of Negative Symptoms (SANS). The University of Iowa, Iowa City.

Andreasen, N.C., 1984. The Scale for the Assessment of Positive Symptoms (SAPS). The University of Iowa, Iowa City.

Andreasen, N.C., Pressler, M., Nopoulos, P., Miller, D., Ho, B.C., 2010. Antipsychotic dose equivalents and dose-years: a standardized method for comparing exposure to different drugs. Biological Psychiatry 67, 255-262.

Anticevic, A., Van Snellenberg, J.X., Cohen, R.E., Repovs, G., Dowd, E.C., Barch, D.M., 2012. Amygdala recruitment in schizophrenia in response to aversive emotional material: a meta-analysis of neuroimaging studies. Schizophrenia Bulletin 38, 608-621.

Brothers, L., 1990. The social brain: A project for integrating primate behavior and neurophysiology in a new domain. Concepts in Neuroscience 1, 27-51.

Brüne, M., 2005. Emotion recognition, 'theory of mind,' and social behavior in schizophrenia. Psychiatry Research 133, 135-147.

Callicott, J.H., Mattay, V.S., Verchinski, B.A., Marenco, S., Egan, M.F., Weinberger D.R., 2003. Complexity of prefrontal cortical dysfunction in schizophrenia: more than up or down. The American Journal of Psychiatry 160, 2209-2215.

Constant, E.L., Lancereau, J., Gillain, B., Delatte, B., Ferauge, M., Bruyer, R., 2011 Deficit in negative emotional information processing in schizophrenia: does i occur in all patients? Psychiatry Research 185, 315-320.

Couture, S.M., Penn, D.L., Roberts, D.L., 2006. The functional significance of social cognition in schizophrenia: a review. Schizophrenia Bulletin 32 (Suppl 1), S44-S63.

Delvecchio, G., Sugranyes, G., Frangou, S., 2013. Evidence of diagnostic specificity in the neural correlates of facial affect processing in bipolar disorder and schizophrenia: a meta-analysis of functional imaging studies. Psychological Medicine 43, 553-569.

Derntl, B., Habel, U., 2011. Deficits in social cognition: a marker for psychiatric disorders? European Archives of Psychiatry and Clinical Neuroscience 261 (Suppl 2), S145-S149.

Dowd, E.C., Barch, D.M., 2010. Anhedonia and emotional experience in schizophrenia: neural and behavioral indicators. Biological Psychiatry 67, 902-911.

Drabant, E.M., McRae, K., Manuck, S.B., Hariri, A.R., Gross, J.J., 2009. Individual differences in typical reappraisal use predict amygdala and prefronta responses. Biological Psychiatry 65, 367-373.

Edwards, J., Jackson, H.J., Pattison, P.E., 2002. Emotion recognition via facia expression and affective prosody in schizophrenia: a methodological review. Clinical Psychology Review Journal 22, 789-832.

Erol, A., Mete, L., Sonmez, I., Unal, E.K., 2010. Facial emotion recognition in patients with schizophrenia and their siblings. Nordic Journal of Psychiatry 64, 63-67.

Esslinger, C., Englisch, S., Inta, D., Rausch, F., Schirmbeck, F., Mier, D., Kirsch, P. Meyer-Lindenberg, A., Zink, M., 2012. Ventral striatal activation during attribution of stimulus saliency and reward anticipation is correlated in unmedicated first episode schizophrenia patients. Schizophrenia Research $140,114-121$.

Gallese, V., 2007. Before and below 'theory of mind': embodied simulation and the neural correlates of social cognition. Philosophical Transactions of the Royal Society of London Series B, Biological Sciences 362, 659-669.

Gardner, D.M., Murphy, A.L., O’Donnell, H., Centorrino, F., Baldessarini, R.J., 2010 International consensus study of antipsychotic dosing. The American Journal of Psychiatry 167, 686-693.

Gradin, V.B., Waiter, G., O'Connor, A., Romaniuk, L., Stickle, C., Matthews, K., Hall, J. Douglas Steele, J., 2013. Salience network-midbrain dysconnectivity and blunted reward signals in schizophrenia. Psychiatry Research: Neuroimaging 211, 104-111.

Habel, U., Chechko, N., Pauly, K., Koch, K., Backes, V., Seiferth, N., Shah, N.J., Stocker, T, Schneider, F., Kellermann, T., 2010. Neural correlates of emotion recognition in schizophrenia. Schizophrenia Research 122, 113-123.

Hall, J., Whalley, H.C., McKirdy, J.W., Romaniuk, L., McGonigle, D., McIntosh, A.M. Baig, B.J., Gountouna, V.E., Job, D.E., Donaldson, D.I., Sprengelmeyer, R., Young, A.W. Johnstone, E.C., Lawrie, S.M., 2008. Overactivation of fear systems to neutral faces in schizophrenia. Biological Psychiatry 64, 70-73.

Haralanova, E., Haralanov, S., Beraldi, A., Moller, H.J., Hennig-Fast, K., 2012 Subjective emotional over-arousal to neutral social scenes in paranoid schizophrenia. European Archives of Psychiatry and Clinical Neuroscience 262, $59-68$.

Hariri, A.R., Bookheimer, S.Y., Mazziotta, J.C., 2000. Modulating emotional responses: effects of a neocortical network on the limbic system. Neuroreport $11,43-48$.

Hempel, A., Hempel, E., Schonknecht, P., Stippich, C., Schroder, J., 2003. Impairment in basal limbic function in schizophrenia during affect recognition. Psychiatry Research: Neuroimaging 122, 115-124.

Holt, D.J., Kunkel, L., Weiss, A.P., Goff, D.C., Wright, C.I., Shin, L.M., Rauch, S.L., Hootnick, J., Heckers, S., 2006a. Increased medial temporal lobe activation during the passive viewing of emotional and neutral facial expressions in schizophrenia. Schizophrenia Research 82, 153-162.

Holt, D.J., Titone, D., Long, L.S., Goff, D.C., Cather, C., Rauch, S.L., Judge, A., Kuperberg, G.R. 2006b. The misattribution of salience in delusional patients with schizophrenia. Schizophrenia Research 83, 247-256.

Jasinska, A.J., Yasuda, M., Rhodes, R.E., Wang, C., Polk, T.A., 2012. Task difficulty modulates the impact of emotional stimuli on neural response in cognitivecontrol regions. Frontiers in Psychology 3, 345.

Keedwell, P.A., Andrew, C., Williams, S.C., Brammer, M.J., Phillips, M.L., 2005. The neural correlates of anhedonia in major depressive disorder. Biological Psychiatry $58,843-853$.

Kiehl, K.A., Smith, A.M., Hare, R.D., Mendrek, A., Forster, B.B., Brink, J., Liddle, P.F, 2001. Limbic abnormalities in affective processing by criminal psychopaths as revealed by functional magnetic resonance imaging. Biological Psychiatry 50, 677-684.

Kohler, C.G., Turner, T.H., Bilker, W.B., Brensinger, C.M., Siegel, S.J., Kanes, S.J., Gur, R.E., Gur, R.C. 2003. Facial emotion recognition in schizophrenia: intensity effects and error pattern. The American Journal of Psychiatry 160, 1768-1774.

Kohler, C.G., Walker, J.B., Martin, E.A., Healey, K.M., Moberg, P.J., 2010. Facial emotion perception in schizophrenia: a meta-analytic review. Schizophrenia Bulletin 36, 1009-1019.

Kosaka, H., Omori, M., Murata, T., Iidaka, T., Yamada, H., Okada, T., Takahashi, T., Sadato, N., Itoh, H., Yonekura, Y., Wada, Y., 2002. Differential amygdala response during facial recognition in patients with schizophrenia: an fMRI study. Schizophrenia Research 57, 87-95.

Laroi, F., Fonteneau, B., Mourad, H., Raballo, A., 2010. Basic emotion recognition and psychopathology in schizophrenia. The Journal of Nervous and Mental Disease 198, 79-81.

Li, H., Chan, R.C., McAlonan, G.M., Gong, O.Y., 2010. Facial emotion processing in schizophrenia: a meta-analysis of functional neuroimaging data. Schizophrenia Bulletin 36, 1029-1039.

Lynch, T.R., Rosenthal, M.Z., Kosson, D.S., Cheavens, J.S., Lejuez, C.W., Blair, R.J., 2006. Heightened sensitivity to facial expressions of emotion in borderline personality disorder. Emotion 6, 647-655.

Malik, F., Khawar, R., Chaudhry, H.R., Humphreys, G.W., 2010. Emotion recognition and duration of untreated first-episode psychosis among patients in Pakistan. East Asian Archives of Psychiatry: Official Journal of the Hong Kong College of Psychiatrists=Dong Ya jing shen ke xue zhi: Xianggang jing shen ke yi xue yuan qi kan 20, 31-38.

Margraf, J., 1994. Mini-DIPS, Diagnostisches Kurz-Interview bei psychischen Störungen. Springer, Berlin.

Mier, D. Lis, S., Esslinger, C., Sauer, C., Hagenhoff, M., Ulferts, J., Gallhofer, B., Kirsch, P., 2013. Neuronal correlates of social cognition in borderline personality disorder. Social Cognitive and Affective Neuroscience 8, 531-537.

Mier, D., Lis, S., Neuthe, K., Sauer, C., Esslinger, C., Gallhofer, B., Kirsch, P., 2010a. The involvement of emotion recognition in affective theory of mind. Psychophysiology 47, 1028-1039.

Mier, D., Sauer, C., Lis, S., Esslinger, C., Wilhelm, J., Gallhofer, B., Kirsch, P., 2010b. Neuronal correlates of affective theory of mind in schizophrenia out-patients: evidence for a baseline deficit. Psychological Medicine 40, 1607-1617.

Mueser, K.T., Doonan, R., Penn, D.L., Blanchard, J.J., Bellack, A.S., Nishith, P., DeLeon, J. 1996. Emotion recognition and social competence in chronic schizophrenia. Journal of Abnormal Psychology 105, 271-275.

Nielsen, M.O., Rostrup, E., Wulff, S., Bak, N., Lublin, H., Kapur, S., Glenthoj, B., 2012. Alterations of the brain reward system in antipsychotic naive schizophrenia patients. Biological Psychiatry 71, 898-905.

Pessoa, L., Padmala, S., Morland, T., 2005. Fate of unattended fearful faces in the amygdala is determined by both attentional resources and cognitive modulation. Neuroimage 28, 249-255.

Pinkham, A.E., Brensinger, C., Kohler, C., Gur, R.E., Gur, R.C., 2011. Actively paranoid patients with schizophrenia over attribute anger to neutral faces. Schizophrenia Research 125, 174-178.

Pinkham, A.E., Hopfinger, J.B., Pelphrey, K.A., Piven, J., Penn, D.L., 2008. Neural bases for impaired social cognition in schizophrenia and autism spectrum disorders. Schizophrenia Research 99, 164-175.

Premkumar, P., Cooke, M.A., Fannon, D., Peters, E., Michel, T.M., Aasen, I., Murray, R.M. Kuipers, E., Kumari, V., 2008. Misattribution bias of threat-related facial expressions is related to a longer duration of illness and poor executive function in schizophrenia and schizoaffective disorder. European Psychiatry 23, $14-19$.

Rasetti, R., Mattay, V.S., Wiedholz, L.M., Kolachana, B.S., Hariri, A.R., Callicott, J.H., Meyer-Lindenberg, A., Weinberger, D.R., 2009. Evidence that altered amygdala activity in schizophrenia is related to clinical state and not genetic risk. The American Journal of Psychiatry 166, 216-225.

Rauch, A.V., Reker, M., Ohrmann, P., Pedersen, A., Bauer, J., Dannlowski, U., Harding, L. Koelkebeck, K., Konrad, C., Kugel, H., Arolt, V., Heindel, W., Suslow, T., 2010. ncreased amygdala activation during automatic processing of facial emotion in schizophrenia. Psychiatry Research: Neuroimaging 182, 200-206.

Rizzolatti, G., Craighero, L., 2004. The mirror-neuron system. Annual Review of Neuroscience 27, 169-192.

Sachs, G., Moser, E., Katschnig, H., Gur, R.C., Gur, R.E., Erfurth, A., 2012. No amygdala attenuation in schizophrenic patients treated with atypical antipsychotics. Psychiatry Research: Neuroimaging 202, 168-171.

Seiferth, N.Y., Pauly, K., Habel, U., Kellermann, T., Shah, N.J., Ruhrmann, S., Klosterkotter, J., Schneider, F., Kircher, T., 2008. Increased neural response 
related to neutral faces in individuals at risk for psychosis. Neuroimage 40, 289-297.

Silver, H., Bilker, W., Goodman, C., 2009. Impaired recognition of happy, sad and neutral expressions in schizophrenia is emotion, but not valence, specific and context dependent. Psychiatry Research 169, 101-106.

Stein, J.L., Wiedholz, L.M., Bassett, D.S., Weinberger, D.R., Zink, C.F., Mattay, V.S., Meyer-Lindenberg, A., 2007a. A validated network of effective amygdala connectivity. Neuroimage 36, 736-745.

Stein, M.B., Simmons, A.N., Feinstein, J.S., Paulus, M.P., 2007b. Increased amygdala and insula activation during emotion processing in anxiety-prone subjects. The American Journal of Psychiatry 164, 318-327.
Taylor, S.F., Kang, J., Brege, I.S., Tso, I.F., Hosanagar, A., Johnson, T.D., 2012. Metaanalysis of functional neuroimaging studies of emotion perception and experience in schizophrenia. Biological Psychiatry 71, 136-145.

Tsoi, D.T. Lee, K.H., Khokhar, W.A., Mir, N.U., Swalli, J.S., Gee, K.A., Pluck, G., Woodruff, P.W., 2008. Is facial emotion recognition impairment in schizophrenia identical for different emotions? A signal detection analysis. Schizophrenia Research 99, 263-269.

Van Snellenberg, J.X., Torres, I.J., Thornton, A.E., 2006. Functional neuroimaging of working memory in schizophrenia: task performance as a moderating variable. Neuropsychology 20, 497-510. 